\title{
Sub-Doppler laser cooling of potassium atoms
}

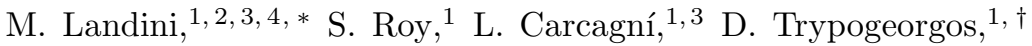 \\ M. Fattori, ${ }^{1,2,3}$ M. Inguscio, ${ }^{1,2,3}$ and G. Modugno ${ }^{1,2,3}$ \\ ${ }^{1}$ LENS and Dipartimento di Fisica e Astronomia, \\ Universitá di Firenze, 50019 Sesto Fiorentino, Italy \\ ${ }^{2}$ INFN, Sezione di Firenze, 50019 Sesto Fiorentino, Italy \\ ${ }^{3}$ INO-CNR, 50019 Sesto Fiorentino, Italy \\ ${ }^{4}$ Dipartimento di Fisica, Università di Trento, 38123 Povo, Trento, Italy
}

(Dated: October 4, 2018)

\begin{abstract}
We investigate sub-Doppler laser cooling of bosonic potassium isotopes, whose small hyperfine splitting has so far prevented cooling below the Doppler temperature. We find instead that the combination of a dark optical molasses scheme that naturally arises in this kind of systems and an adiabatic ramping of the laser parameters allows to reach sub-Doppler temperatures for small laser detunings. We demonstrate temperatures as low as $(25 \pm 3) \mu \mathrm{K}$ and $(47 \pm 5) \mu \mathrm{K}$ in high-density samples of the two isotopes ${ }^{39} \mathrm{~K}$ and ${ }^{41} \mathrm{~K}$, respectively. Our findings will find application to other atomic systems.
\end{abstract}

PACS numbers: $37.10 . \mathrm{De} ; 37.10 . \mathrm{Vz}$

Sub-Doppler laser cooling of neutral atoms [1] is a key technique for the production of ultracold and quantum gases. It allows for atoms to be cooled to temperatures below the Doppler limit [2], not far from the single photon recoil energy. This favors the application of further cooling techniques, such as Raman or evaporative cooling, to reach quantum degeneracy. It also realizes a fast and effective cooling method for some classes of atomic interferometers and clocks [3]. The sub-Doppler cooling mechanism arises whenever the atomic ground state has an internal structure with state-dependent light shifts. Such a situation is typically accompanied by a hyperfine structure of the excited state [4]. The sub-Doppler cooling is efficient only if the excited state has a hyperfine splitting $\Delta$ either much larger than the natural linewidth $\Gamma$, like that for the alkalis $\mathrm{Na}, \mathrm{Rb}$ and $\mathrm{Cs}$, or smaller than $\Gamma$, as for example in $\mathrm{Sr}[5]$. In the intermediate case of $\Delta \sim \Gamma$, it can instead be hindered by the presence of heating forces or by photon reabsorption [6]. The bosonic potassium isotopes fall into this latter category [7], and no efficient sub-Doppler cooling has been observed so far [8-13].

We now instead find that sub-Doppler cooling can take place also in atoms like $\mathrm{K}$, by employing a near-detuned optical molasses and an appropriate strategy to tune the cooling laser parameters. We observe that the natural depumping towards dark states taking place in this kind of systems allows us to reach low temperatures even in high-density samples. In experiments on the isotopes ${ }^{39} \mathrm{~K}$ and ${ }^{41} \mathrm{~K}$, we achieve temperatures substantially lower than those previously achieved, with an efficiency similar to that of most other alkali species.

In a two-level system, Doppler cooling arises when a laser is tuned below the atomic transition frequency, where the atoms experience a friction force: $F=-\alpha v$. If the presence of Zeeman sub-levels is taken into ac-
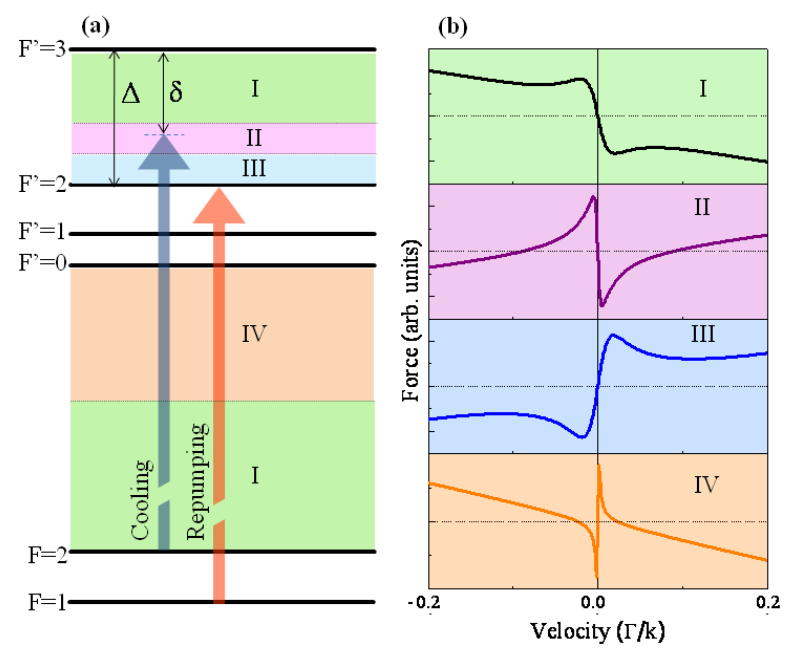

FIG. 1: Working regions for sub-Doppler cooling of bosonic Potassium. a) Level scheme including the relevant hyperfine splitting $\Delta$ and the cooling laser detuning $\delta$. b) Calculated cooling forces vs the atomic velocity in the various regions of (a). Doppler cooling only takes place in regions I and IV, while sub-Doppler cooling is active only in regions I and II.

count [2], a much larger friction arises for small velocities, leading to temperatures much lower than the Doppler limit $k_{B} T_{D}=\hbar \Gamma / 2$. While in principle, the lowest achievable sub-Doppler temperatures are independent of the laser detuning $\delta$ [15], the experiments with large density samples are performed at large detunings, $\delta \gg \Gamma$. This requirement arises from the need of keeping the scattering rate of photons by individual atoms low, in such a way that spontaneously emitted photons may not disturb the cooling process [6]. Most atomic systems cannot be modeled as simple two-level ones since they feature a hyperfine structure like the one in Fig. 1 that is 
relevant for instance to $\mathrm{Na}, \mathrm{K}$ and $\mathrm{Rb}$. In this case, it is commonly thought that $\delta$ must also be smaller than the main hyperfine splitting $\Delta$, since otherwise the presence of the other excited states would turn the sub-Doppler mechanism into a heating one. As a matter of fact, in the case of the bosonic $\mathrm{K}$ isotopes, where $\Delta \approx 2 \Gamma-3 \Gamma$, a clear sub-Doppler cooling has not been experimentally observed. This can be understood from the nature of the optical forces we have calculated for the level structure in Fig, 1, sub-Doppler cooling is active either very close to resonance, where heating from photon reabsorption might be large, or for $\delta \gg \Delta$, where however the velocity capture range is very low. Note that in the case of $\Gamma>\Delta$, such as for ${ }^{87} \mathrm{Sr}$, the sub-Doppler cooling stays efficient also for $\delta>\Delta[5]$.

We have now realized that the presence of neighboring excited states, however, has also a beneficial effect. Indeed it causes a natural depumping of the atomic population into a dark state, such as the $F=1$ ground state as shown in Fig. 1. The atoms can of course be moved back into the bright $F=2$ state by the repumper laser but, differently from a pure two-level system, this can be done in a controlled way. It is then possible to adjust the fraction of atoms in the state coupled to the cooling laser in order to optimize the cooling power, while keeping the reabsorption of spontaneously emitted photons under control. Note that the possibility of controlling the population of the bright state is absent if $\Delta \gg \Gamma$ unless an appropriate depumping laser is used [16]. This mechanism, which is widely used to trap atomic samples at high-density in magneto-optical traps [17], turns out to be the first essential ingredient for sub-Doppler cooling when $\Delta \approx \Gamma$, since it allows to reach low temperatures also when $\delta \approx \Gamma$. This is apparent from Fig 2, which shows the minimum temperature we measured for ${ }^{39} K$ in near-resonant molasses with a very low intensity of the repumping light and a large atomic density (further details are given below). The measured temperature is well below the Doppler limit already for $\delta<\Gamma$, although a further decrease with increasing $\delta$ is apparent.

The second important observation is that the subDoppler cooling survives for detunings larger than the Doppler cooling does, as shown for example in the calculations of Fig 1. The lowest temperatures can actually be reached only for a range of detunings where the Doppler force does no longer provide an efficient cooling. This is in principle a problem in experiments, since the velocity capture range of the sub-Doppler cooling mechanism is usually smaller than the initial thermal velocity, for example at the end of the capture stage of a magnetooptical trap. Indeed, one normally needs to exploit both Doppler and sub-Doppler cooling to achieve low temperatures 2]. We now find that one can still combine an initial Doppler cooling with a final stage of optimal subDoppler cooling by using a proper dynamical variation of detuning and intensity of the cooling laser between the

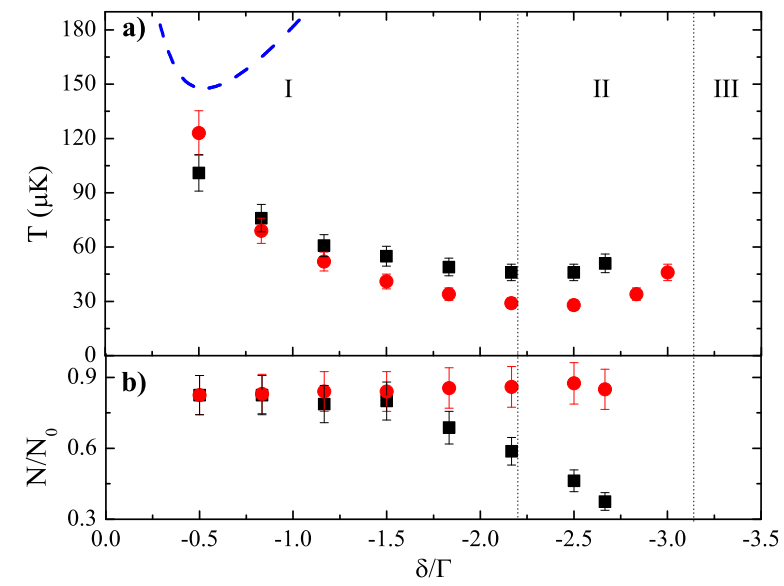

FIG. 2: Optimal sub-Doppler temperatures for ${ }^{39}$ K. a) Measured temperature without (black squares) and with (red dots) the ramping strategy compared to the Doppler theory (dashed line). The dotted lines separate the various regions as in Fig. 1, b) Fraction of atoms remaining in the colder component without (black squares) and with (red dots) the ramping strategy.

two regimes of operation. As a matter of fact, the combination of these two ingredients makes the cooling of $\mathrm{K}$ as efficient as in the other alkali species.

We now discuss in detail the experimental strategy. The linewidth of the cooling transition for $\mathrm{K}$ is $\Gamma=$ $2 \pi \times 6.0 \mathrm{MHz}$, which corresponds to a Doppler temperature $T_{D} \approx 145 \mu \mathrm{K}$. The hyperfine splitting $\Delta$ is about $3.5 \Gamma$ and $2.2 \Gamma$ for ${ }^{39} \mathrm{~K}$ and ${ }^{41} \mathrm{~K}$ respectively. We perform cooling and trapping in a three-dimensional magnetooptical trap (MOT) on the $D_{2}$ transition around $767 \mathrm{~nm}$. The trap is loaded with pre-cooled atoms from a twodimensional MOT. After $3 \mathrm{~s}$ of loading stage we have either about $2 \times 10^{10}$ atoms of ${ }^{39} \mathrm{~K}$ or $4 \times 10^{9}$ atoms of ${ }^{41} \mathrm{~K}$ at temperatures in the $1 \mathrm{mK}$ regime. We then compress the cloud via application of a compressed-MOT technique to densities around $1 \times 10^{11}$ atoms $/ \mathrm{cm}^{3}$. During this initial cooling stage we adopt the standard strategy used for bosonic potassium 9, 13. We use a detuning larger than the whole excited manifold (region IV) with total intensities as large as $20 I_{s}$ for both the cooling $\left(\mathrm{I}_{\text {cool }}\right)$ and the repumping $\left(\mathrm{I}_{r e p}\right)$ beams $\left(I_{s}=1.75 \mathrm{~mW} / \mathrm{cm}^{2}\right)$. This allows for a large Doppler capture velocity. Finally, we switch off the magnetic field and we cool the cloud in a molasses scheme as described below.

We initially reduce suddenly $\mathrm{I}_{\text {rep }}$ to $1 / 100 \mathrm{I}_{\text {cool }}$ and we set the repumper beam frequency on resonance with the $F=1 \rightarrow F^{\prime}=2$ transition. If we then try to perform standard molasses cooling, i.e. by a sudden change of the laser parameters to the optimal sub-Doppler cooling values, we observe moderate sub-Doppler cooling only for small $\delta$. A larger $\delta$ results instead in a bimodal distribution of the atomic velocities. A typical instance of such 

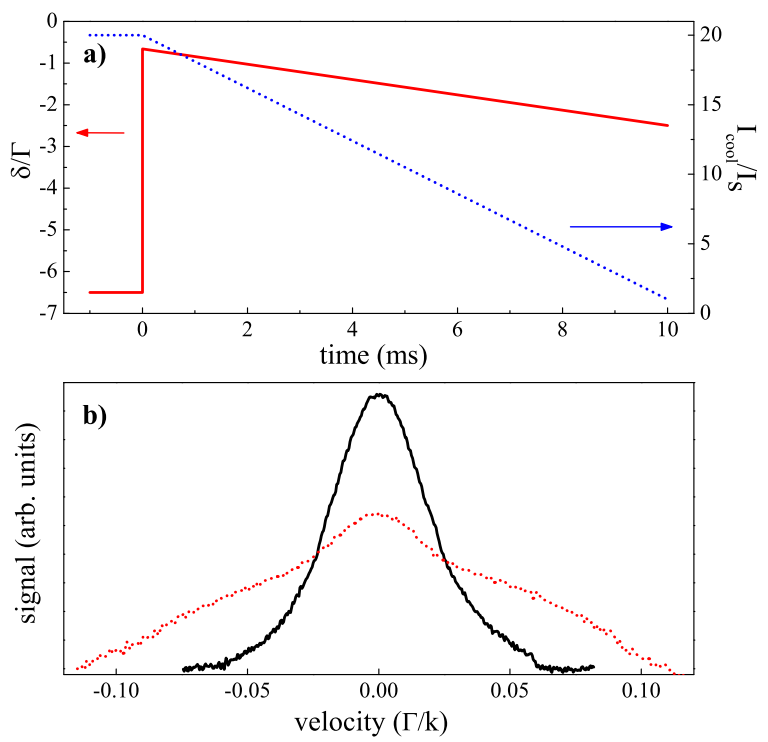

FIG. 3: Sub-Doppler cooling strategy for ${ }^{39} \mathrm{~K}$. a) Time evolution of $\mathrm{I}_{\text {cool }}$ and $\delta\left(\mathrm{I}_{r e p}=1 / 100 \mathrm{I}_{\text {cool }}\right)$. b) Resulting velocity distribution measured after a free expansion (black solid) compared to that obtained without the ramp (red dashed).

distribution is shown in Fig. 3b, as measured by fluorescence imaging after a free expansion of the cloud [18]. The narrow peak corresponds to sub-Doppler temperatures, while the broader distribution can be attributed to an inefficient Doppler cooling or even to a Doppler heating. As shown in Fig. 2 the fraction of atoms in the central component decreases as $\delta$ is increased. A more effective strategy consists in first tuning the laser to $\delta \approx \Gamma / 2$ to provide an initial Doppler cooling and then in slowly decreasing the intensity while increasing $\delta$, as shown in Fig. 3 a. This method allows to cool nearly $90 \%$ of the atoms to lower temperatures, as shown in Fig. 2 . By minimizing the final temperature we find an optimal ramping time of about $10 \mathrm{~ms}$, which corresponds to an adiabatic narrowing of the velocity distribution during the whole sequence. The minimum temperature attained for ${ }^{39} \mathrm{~K}$ is about $25 \mu \mathrm{K}$ at $\delta \approx 2.5 \Gamma$. It then rises again for larger $\delta$, presumably because of the progressive reduction of the force.

We have observed, as shown in Fig. 4, that an increase of the repumping power prevents the achievement of such low temperatures at high density, while the temperature does not depend on the repumper power at low density. This confirms the role of reabsorption of spontaneously emitted photons inside the cloud.

We have performed analogous measurements on ${ }^{41} \mathrm{~K}$ and found a similar behavior. In this case a minimum temperature of about $50 \mu \mathrm{K}$ is reached for a detuning $\delta \approx \Gamma$. We have compared the observations with a theoretical estimation of the temperatures achievable for our experimental parameters. The optical force, shown in

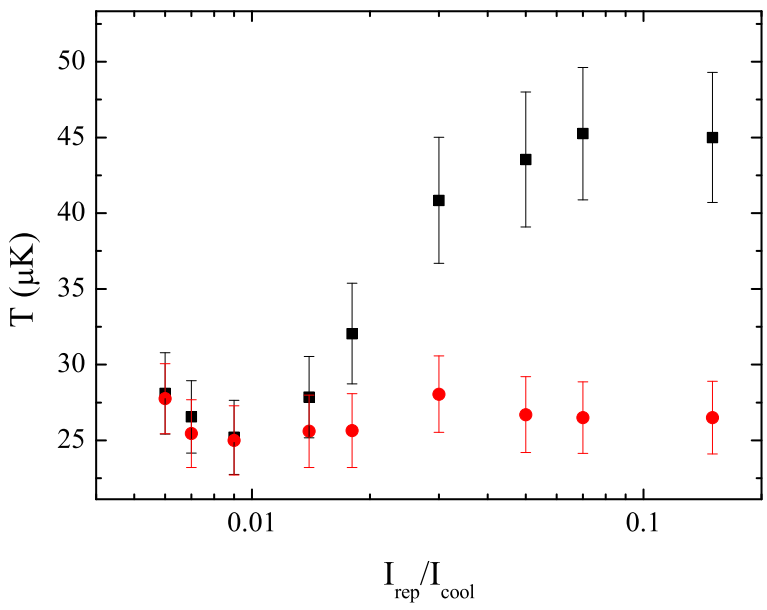

FIG. 4: Measured temperatures for ${ }^{39} \mathrm{~K}$ vs the intensity ratio of repumping and cooling light, for densities of $4 \times 10^{10}$ atoms $/ \mathrm{cm}^{3}$ (black squares) and $8 \times 10^{8}$ atoms $/ \mathrm{cm}^{3}$ (red dots). The heating arising from reabsorption effects at high density can be tuned by reducing the repumper intensity.

Fig. 1, is calculated from the solution of the optical Bloch equations in the semi-classical approximation [7, 9]. The model is only accurate in $1 \mathrm{D}$ and for $\sigma^{+}-\sigma^{-}$polarizations [18]. The agreement with the experimental data

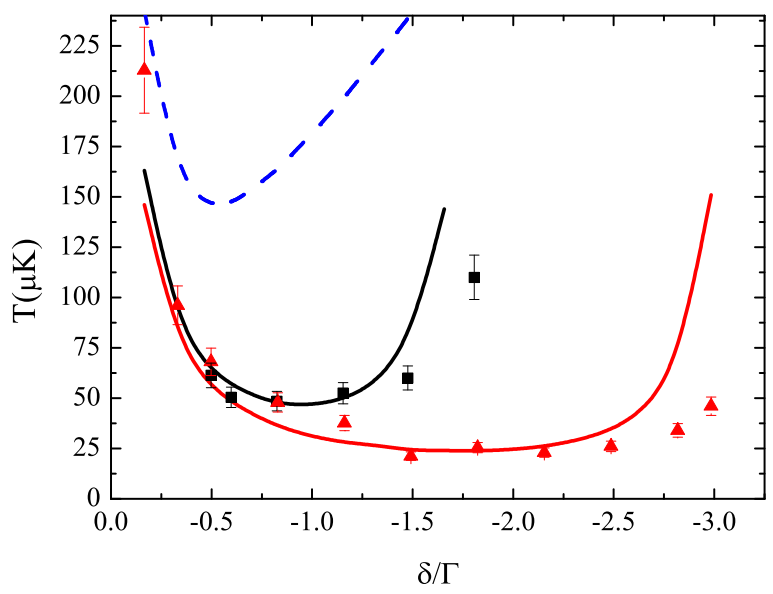

FIG. 5: Measured temperatures for ${ }^{39} \mathrm{~K}$ (red triangles) and ${ }^{41} \mathrm{~K}$ (black squares) and calculated temperatures (lines) vs the cooling laser detuning. The dashed line is the predicted Doppler temperature.

shown in Fig. [5] is however rather good. To check the validity of these calculations we directly measured the spatial diffusion coefficient $D_{x}$ in the optical molasses for ${ }^{39} \mathrm{~K}$ [18]. By a simultaneous measurement of the temperature we estimated the friction coefficient as $\alpha=k_{B} T / D_{x}[1]$. The magnitude of $\alpha \approx 10^{-3} \hbar k^{2}$ is in good agreement with the calculations. A simple estimation considering a two-level system would give a result about 3 orders of 
magnitude larger. We interprete this low friction as a result of the macroscopical occupation of the dark state.

The minimum temperatures at each detuning in Fig. 5 were obtained for an almost constant laser intensity. The data at small detunings is apparently well described by the scaling law observed in several other systems [5, 2023]:

$$
T=C_{\sigma^{+} \sigma^{-}} \frac{\hbar \Gamma}{2 k_{B}} \frac{\Gamma}{|\delta|} \frac{I}{I_{s}}+T_{0} .
$$

From a combined fit we get $C_{\sigma^{+} \sigma^{-}}=0.20(2)$ and $T_{0}=$ $9(3) \mu \mathrm{K}$. The $C_{\sigma^{+} \sigma^{-}}$coefficient is smaller than those measured on the two species where sub-Doppler cooling has been observed for $\delta \lesssim \Gamma$, i.e. ${ }^{87} \operatorname{Er}[22](0.38(2))$ and ${ }^{87} \mathrm{Sr}$ [5] $(1.3(3))$ but larger than those measured in $\mathrm{Rb}$ and Cs at large detunings [21]. The observed scaling for $\mathrm{K}$ suggests that heating processes due to reabsorption are less efficiently suppressed when $\delta$ is small, as expected.

To characterize the robustness of the cooling process against stray magnetic fields we tried to keep the MOT magnetic field on during the cooling procedure. For gradients larger than $5 \mathrm{G} / \mathrm{cm}$, we reached the Doppler temperature. This gradient corresponds to an average magnetic field of about $1 \mathrm{G}$, which is the same characteristic value found for the other alkali atoms.

The techniques described here might be applied to other systems, such as the ${ }^{1} \mathrm{~S} \rightarrow{ }^{1} \mathrm{P}$ transitions of ${ }^{43} \mathrm{Ca}$ [24] and ${ }^{173} \mathrm{Yb}[25]$, for which $\Delta \approx 3 \Gamma$. Additionally, it would be interesting to apply our cooling strategy to $\mathrm{Na}$, for which $\Delta \approx 6 \Gamma$. Another interesting case is that of an inverted and narrow hyperfine structure like in ${ }^{40} \mathrm{~K}$. In this case, there are no interfering levels which can directly cause heating. However, an increase of $\delta$ to values of the order of $\Delta$ or more leads to a washing out of the sub-Doppler cooling mechanism itself, since the detuning from the various hyperfine levels becomes of the same order. We performed numerical simulations of the atomic force and we found that this effect leads to a fast decrease of the capture velocity with increasing detuning [18]. This decrease is faster than the one for atoms with a large $\Delta$ (or with a large $\Gamma / \Delta$ as in the case of $\mathrm{Sr}$ ), and leads to a regime in which the minimum subDoppler temperature $k_{B} T=D_{p} / \alpha$ exceeds the capture range. This is presumably the reason for the bimodal distribution seen in experiments with ${ }^{40} K[26]$. Weaker rates of natural depumping to dark states will possibly require forced depumping [16] in high density samples, but further experimental and numerical investigations are needed [27].

We have shown how the limitations of sub-Doppler laser cooling in atomic species with small hyperfine splitting can be overcome by the natural control of the photon reabsorption and adiabatic ramping of the laser parameters. The laser cooling techniques we have developed will be easily implemented in all existing experiments with potassium atoms. Finally, the direct application of laser cooled potassium atoms to interferometric measurements might enable a new class of experiments [28].

Note: we recently learned of another report of subDoppler temperatures in K [29].

We gratefully acknowledge contributions by G. Roati, S. Ferrari and F. Cataliotti. This work is dedicated to the memory of Arturo Bambini who contributed with his deep insight to the early investigations on ultracold potassium in Florence. This work was supported by INFN (MICRA collaboration), by EU (IP AQUTE), by ERC (DISQUA and QUPOL grants) and by the ESF and CNR (EuroQUASAR program).

* Electronic address: landini@science.unitn.it

† Present address: Clarendon Laboratory, University of Oxford, Parks Road, Oxford, OX1 3PU, UK

[1] S. Chu, Rev. Mod. Phys. 70, 685 (1998); C. Cohen- Tannoudji, ibid. 70, 707 (1998); W. D. Phillips, ibid. 70, 721 (1998).

[2] T. W. Hänsch and A. L. Schawlow, Opt. Comm. 13, 68 (1975).

[3] C. Bord, Metrologia , 39, 435 (2002).

[4] E. Arimondo, M. Inguscio and P. Violino, Rev. Mod. Phys. 49, Vol. 1, 31 (1977).

[5] X. Xu, T. H. Loftus, J. W. Dunn, C. H. Greene, J. L. Hall, A. Gallagher, and J. Ye, Phys. Rev. Lett. 90, 193002 (2003).

[6] C. J. Cooper, G. Hillenbrand, J. Rink, C. G. Townsend, K. Zetie and C. J. Foot, Europhys. Lett. 28, 397 (1994).

[7] A. Bambini, and A. Agresti, Phys. Rev. A 56, 3040 (1997).

[8] R. S. Williamson III and T. Walker, J. P, J. Opt. Soc. Am. B 1213931393 (1995).

[9] C. Fort, A. Bambini, L. Cacciapuoti, F. S. Cataliotti, M. Prevedelli, G. M. Tino, and M. Inguscio, Eur. Phys. J. D 3, 113 (1998).

[10] M. Prevedelli, F.S. Cataliotti, E.A. Cornell, J.R. Ensher, C. Fort, L. Ricci, G.M. Tino, and M. Inguscio, Phys. Rev. A 59, 886 (1999).

[11] L. De Sarlo, P. Maioli, G. Barontini, J. Catani, F. Minardi and M. Inguscio, Phys. Rev. A 75, 022715 (2007).

[12] G. Modugno, G. Ferrari, G. Roati, R. J. Brecha, A. Simoni and M. Inguscio, Science 294, 1320 (2001).

[13] T. Kishimoto, J. Kobayashi, K. Noda, K. Aikawa, M. Ueda, and S. Inouye,Phys. Rev. A 79, 031602(R) (2009).

[14] J. Dalibard, and C. Cohen-Tannoudji, J. Opt. Soc. Am B. 6, 2023 (1989).

[15] J. Dalibard and Y. Castin, in the Frontiers in Laser Spectroscopy, proceedings of the CXX International School of Physics Enrico Fermi edited by T.W. Hänsch and M. Inguscio (North- Holland, 1994).

[16] C. G. Townsend, N. H. Edwards, K. P. Zetie, C. J. Cooper, J. Rink, and C. J. Foot, Phys. Rev. A 53, 1702 (1996).

[17] W. Ketterle, K. B. Davis, M. A. Joffe, A. Martin and D. E. Pritchard, Phys. Rev. Lett., 70, 2253 (1993).

[18] See the supplementary material for further details.

[19] T. W. Hodapp, C. Gerz, C. Furtlehner, C. I. Westbrook, W. D. Phillips, J. Dalibard, Appl. Phys. B 60, 135 (1995).

[20] C. Gerz, T.W. Hodapp, P. Jessen, K.M. Jones, W.D. 
Phillips, C.I. Westbrook, and K. Mo.lmer, Europhys. Lett. 21, 661 (1993).

[21] Y. Castin, and K. Mølmer, Phys. Rev. Lett. 74, 3772 (1995).

[22] A. J. Berglund, S. A. Lee, and J. J. McClelland, Phys. Rev. A 76, 053418 (2007).

[23] R. Maruyama, R. H. Wynar, M. V. Romalis, A. Andalkar, M. D. Swallows, C. E. Pearson, and E. N. Fortson, Phys. Rev. A 68, 011403(R) (2003).

[24] A. Mortensen, J. J. T. Lindballe, I. S. Jensen, P. Staanum, D. Voigt, and M. Drewsen, Phis. Rev. A, 69, 042502 (2004).

[25] T. Kuwamoto, K. Honda, Y. Takahashi, and T. Yabuzaki, Phys. Rev. A 60, R745 (1999).

[26] G. Modugno, C. Benko, P. Hannaford, G. Roati, and M. Inguscio, Phys. Rev. A 60, R3373 (1999).

[27] Josh W. Dunn and Chris H. Greene, Phys. Rev. A 73, 033421 (2006).

[28] G. Varoquaux, R. A. Nyman, R. Geiger, P. Cheinet, A. Landragin and P. Bouyer, New. J. Phys. 11113010 (2009)

[29] V. Gokhroo, G. Rajalakshmi, R. K. Easwaran and C. S. Unnikrishnan, J. Phys. B: At. Mol. Opt. Phys. 44, 115307 (2011).

\section{SUPPLEMENTARY MATHERIAL}

\section{TEMPERATURE MEASUREMENT}

Here we give a brief description of our detection strategy. We image the atomic cloud by florescence imaging after time of flight. To do so we release the trap and after a variable time we pulse the MOT beams at the maximum intensity for $30 \mu s$ (the high intensity ensures the saturation of the atomic transition). We collect the scattered light with an imaging system on a CCD camera. Due to the high optical density of the sample, images taken on resonance showed saturation effects and hence prevented us from estimating the correct number of atoms. Hence we detune the lasers on the blue of the $\mathrm{F}=2 \rightarrow \mathrm{F}^{\prime}=3$ transition. Detuning to the red side of the transition was avoided due to the proximity of the other hyperfine energy levels. To determine the number of atoms by this method we first perform spectroscopy on the atomic cloud at low density and at the usual intensity recording the power broadening. To check the validity of this technique we also try to perform temperature measurements tuning the laser on resonance. Although the width of the cloud was affected by saturation effects, the resulting temperatures were consistent with the ones measured while detuning the laser out of resonance. We fit the atomic signal with a 2D Gaussian distribution to extract the $\mathrm{e}^{-1 / 2}$ half-width $\sigma$ of the distribution, measuring it at different expansion times and henceforth we can reconstruct $\sigma\left(t_{\text {exp }}\right)$, which we fit by the equation

$$
\sigma\left(t_{\text {exp }}\right)^{2}=\sigma_{0}^{2}+\frac{k_{B} T}{m} t_{\text {exp }}^{2} .
$$

From the results of the fit we extract the temperature

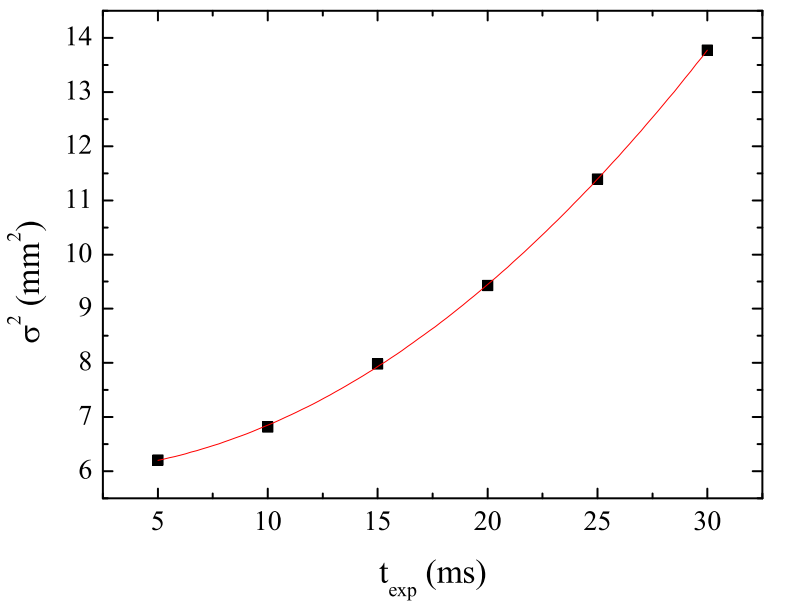

FIG. 6: Typical evolution of the atomic distribution width as a function of time after release from the molasses cooling beams. The solid line is a fit by Eq. 2 .

as well as the initial size of the cloud, which is used in order to measure the density of the atomic cloud.

In order to precisely measure the magnification of our imaging system we use two different methods. The first one consists of loading the atoms into a magnetic trap mounted on a translation stage. The positioning of the translation stage has $10 \mu \mathrm{m}$ accuracy. By movement by a given amount of the coils and recording the position of the center of the cloud on the CCD, we are able to measure the magnification, which is found to be: 1/3.23(1). The second method consists of allowing the atomic cloud to fall due to gravity after switching off the trap and recording its position as a function of time. From the fit of the position by the law of free fall and assuming for the gravitational acceleration: $\mathrm{g}=9.81 \mathrm{~m} / \mathrm{s}^{2}$ we find the value of the magnification to be $1 / 3.22(5)$, which is in good agreement with the value determined by the other method.

In case of the bimodal distributions as in Fig. 2 of the main paper, we fit just the cold component after a long time of flight ( $\left.\mathrm{t}_{\exp }>10 \mathrm{~ms}\right)$.

\section{DIFFUSION MEASUREMENT}

We measure the spatial diffusion coefficient $\mathrm{D}_{x}$ for the Brownian atomic motion during the molasses cooling phase. This is a useful quantity since it connects to the other quantities characterizing the cooling process (namely $\mathrm{T}, \alpha$ and $\mathrm{D}_{p}$ ) via the simple formula:

$$
D_{x}=\frac{D_{p}}{\alpha^{2}}=\frac{k_{B} T}{\alpha} .
$$

Hence, by measuring both $\mathrm{T}$ and $\mathrm{D}_{x}$ one can reconstruct the other useful quantities. The measurement is performed at different final detuning of the molasses cooling 


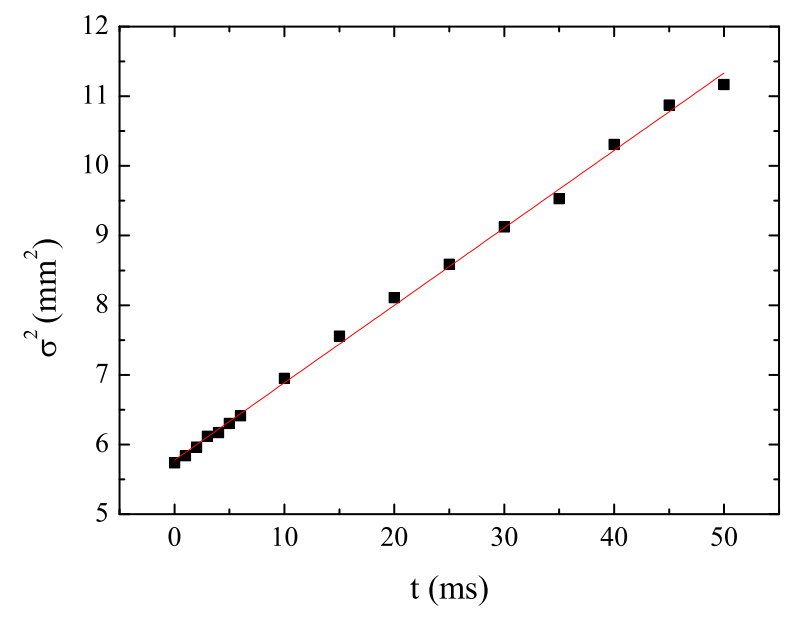

FIG. 7: Typical data for the variation of the width of the atomic distribution as a function of the time spent inside the molasses cooling beams. The solid line is a fit by Eq. 3 .

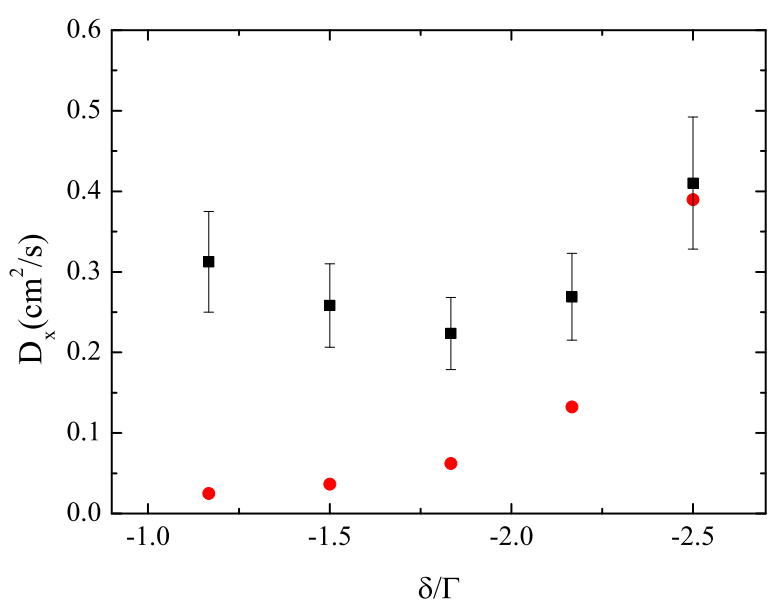

FIG. 8: Measured diffusion coefficients in the optical molasses (black squares) compared to the numerically calculated values (red dots) as a function of the detuning of the cooling laser.

beams by allowing the atomic cloud to expand in presence of the cooling light for a variable time, and then taking an image after $100 \mu$ s dark period. By recording the variation of the width with time in the molasses cooling beams, we find it to be in agreement with a diffusion process (Fig. 7). By fitting the evolution of the cloud size with the equation:

$$
\sigma(t)^{2}=\sigma_{0}^{2}+2 D_{x} t
$$

we are able to extract the spatial diffusion coefficient. From the results in Fig. 8 we see that the agreement with the theory is good for our usual experimental parameters but the agreement becomes rather poor for smaller detunings. This might be due to the presence of additional heating from rescattered photons since these measure- ments were taken at high densities $\left(4 \times 10^{10}\right.$ atoms $\left./ \mathrm{cm}^{3}\right)$. These values for the diffusion coefficient are a factor about of 1000 times higher than the ones measured in [1]. The reason for this is the very low population in the $\mathrm{F}=2$ level caused by natural depumping and the use of a very weak repumping light.

\section{NUMERICAL SIMULATION}

We perform numerical simulations of the optical Bloch equations for multilevel systems to find the optical forces acting on the atoms during the molasses cooling. We work in $1 \mathrm{D}$ and in the $\sigma_{+}-\sigma_{-}$configuration for the laser beams polarizations. This is only an approximation to the more complex polarization geometries arising in the 3D laser configuration we have in our experiment. In practical situations, in a magneto-optical trap, both Sisyphus cooling (lin $\perp$ lin) and $\sigma_{+}-\sigma_{-}$polarization gradient cooling play a role in the cooling process, with the former dominating at large detuning and the latter at small detuning [2, 3]. In our simulation, only the cooling beam is taken into account to find the cooling force. This is justified by the very low repumper intensity we use in the experiment (about $10^{-2} \mathrm{I}_{s}$ ).

In the determination of the different regions of Fig. 1 of the main paper we focus our attention on a velocity range corresponding to twice the width of our initial velocity distribution (just before the molasses phase the atomic temperature is about $1 \mathrm{mK}$ ) and we set the cooling laser intensity to be $\mathrm{I}_{s}$. The cooling force was calculated as a function of velocity for different detunings. The whole range of detunings for which the force was calculated was divided into four regions. Region I: This region comprises of the detuning values for which the force is opposite to velocity for the whole velocity range and hence always provides cooling. Region II: The force is opposite to the velocity only for small velocities, whereas for higher velocities the force changes sign, thereby providing heating. Region III: In this region, the force provides heating in the whole velocity range. Region IV: The force provides heating for low velocities, whereas for higher velocities the force is opposite to the velocity. In Fig. 1 of the main paper we do not report the force behavior for detuning values in between the $\mathrm{F}^{\prime}=2$ and $\mathrm{F}^{\prime}=0$ levels.

The calculated temperatures of Fig.5 in the main paper are calculated as $k_{B} T=D_{p} / \alpha$ where $D_{p}$ is the momentum diffusion coefficient and $\alpha$ is the friction coefficient calculated as the slope of the force at $v=0$. We estimate $D_{p}$ by using the simple argument for the random step of the Brownian motion in momentum space in multilevel transitions described in Ref. [4].

To compare the efficiency of the cooling process in the case of narrow hyperfine structure to the two-level case we numerically simulate the cooling force and extract the velocity capture range $\mathrm{v}_{c}$, defined as the velocity giving 


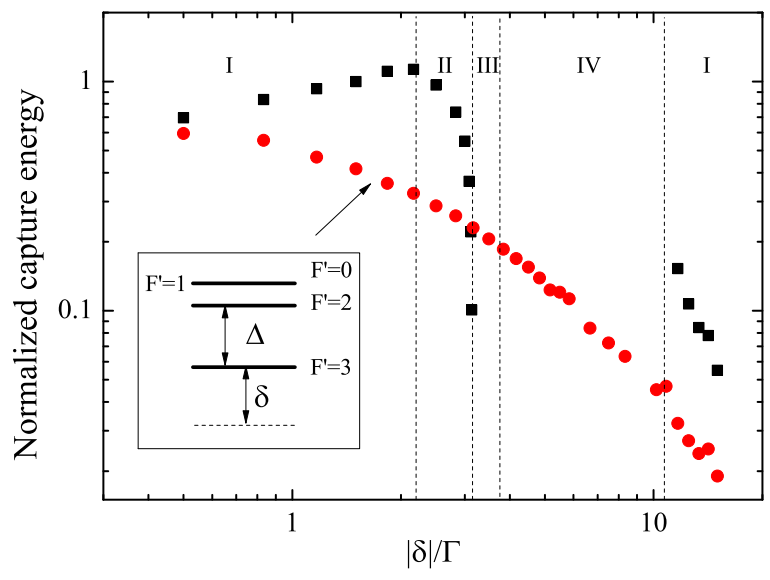

FIG. 9: Calculated sub-Doppler capture energy for ${ }^{39} \mathrm{~K}$ (black squares) and for a hypothetical atomic species with an inverted hyperfine structure as shown in the inset (red dots). Both quantities are normalized to the capture energy of an atomic species with $\Delta=35 \Gamma$.

the first local maximum of force at low velocity. We do this in three cases: a) a hyperfine level structure like the one of ${ }^{39} \mathrm{~K}, \mathrm{~b}$ ) an equal but inverted hyperfine level structure, c) the same hyperfine structure but with a 10-fold increase in the hyperfine splitting. In Fig 9 we plot the capture energy $E_{c}=\frac{1}{2} m v_{c}^{2}$ for the case of ${ }^{39} \mathrm{~K}$ normalized by the 10 -fold increased case (black squares), and the inverted case normalized by the same quantity (red dots). We show only the value of $\mathrm{E}_{C}$ in the detuning regions in which the sub-Doppler cooling mechanism provides cooling (region I and II of Fig.1 in the main paper). This fast decrease of the velocity capture range makes the achievement of sub-Doppler cooling difficult for optical molasses at large detuning for this kind of systems. In conclusion, the numerical simulation provides a good understanding of the sub-Doppler cooling process and the results obtained in our experiment.

* Electronic address: landini@science.unitn.it

$\dagger$ Present address: Clarendon Laboratory, University of Oxford, Parks Road, Oxford, OX1 3PU, UK

[1] T. W. Hodapp, C. Gerz, C. Furtlehner, C. I. Westbrook, W. D. Phillips, J. Dalibard, Appl. Phys. B 60, 135 (1995).

[2] J. Dalibard, and C. Cohen-Tannoudji, J. Opt. Soc. Am B. 6, 2023 (1989).

[3] S. A. Hopkins and A. V. Durrant, Phys. Rev. A 56, 4012 (1997).

[4] Y. Castin, and K. Mølmer, J. Phys. B: At. Mol. Opt. Phys. 234101 (1990). 